\title{
Meta-analysis identifies five novel loci associated with endometriosis highlighting key genes involved in hormone metabolism
}

Yadav Sapkota ${ }^{1,2}$, Valgerdur Steinthorsdottir ${ }^{3}$, Andrew P. Morris ${ }^{4,5}$, Amelie Fassbender $^{6,7}$, Nilufer Rahmioglu ${ }^{5}$, Immaculata De Vivo ${ }^{8,9}$, Julie E. Buring ${ }^{8,10}$, Futao Zhang ${ }^{11}$, Todd L. Edwards ${ }^{12}$, Sarah Jones ${ }^{13}$, Dorien O ${ }^{6,7}$, Daniëlle Peterse ${ }^{6,7}$, Kathryn M. Rexrode ${ }^{8,10}$, Paul M. Ridker ${ }^{8,10}$, Andrew J. Schork ${ }^{14,15}$, Stuart MacGregor', Nicholas G. Martin', Christian M. Becker ${ }^{16}$, Sosuke Adachi ${ }^{17}$, Kosuke Yoshihara ${ }^{17}$, Takayuki Enomoto ${ }^{17}$, Atsushi Takahashi ${ }^{18}$, Yoichiro Kamatani ${ }^{18}$, Koichi Matsuda ${ }^{19}$, Michiaki Kubo ${ }^{18}$, Gudmar Thorleifsson', Reynir T. Geirsson ${ }^{20,21}$, Unnur Thorsteinsdottir ${ }^{3,21}$, Leanne M. Wallace ${ }^{1,11}$, iPSYCH-SSI-Broad Group ${ }^{\dagger}$, Jian Yang ${ }^{11}$, Digna R. Velez Edwards ${ }^{22}$, Mette Nyegaard ${ }^{23,24}$, Siew-Kee Low ${ }^{18, \star}$, Krina T. Zondervan ${ }^{5,16, \star}$, Stacey A. Missmer ${ }^{8,9, *}$, Thomas D'Hooghe ${ }^{6,7,25, \star}$, Grant W. Montgomery ${ }^{1,11, *}$, Daniel I. Chasman $8,10, \star$, Kari Stefansson ${ }^{3,21, \star}$, Joyce Y. Tung ${ }^{26, \star} \&$ Dale R. Nyholt ${ }^{1,27, \star}$

Endometriosis is a heritable hormone-dependent gynecological disorder, associated with severe pelvic pain and reduced fertility; however, its molecular mechanisms remain largely unknown. Here we perform a meta-analysis of 11 genome-wide association case-control data sets, totalling 17,045 endometriosis cases and 191,596 controls. In addition to replicating previously reported loci, we identify five novel loci significantly associated with endometriosis risk $\left(P<5 \times 10^{-8}\right)$, implicating genes involved in sex steroid hormone pathways (FN1, CCDC170, ESR1, SYNE1 and FSHB). Conditional analysis identified five secondary association signals, including two at the ESR1 locus, resulting in 19 independent single nucleotide polymorphisms (SNPs) robustly associated with endometriosis, which together explain up to 5.19\% of variance in endometriosis. These results highlight novel variants in or near specific genes with important roles in sex steroid hormone signalling and function, and offer unique opportunities for more targeted functional research efforts.

\footnotetext{
${ }^{1}$ Department of Genetics and Computational Biology, QIMR Berghofer Medical Research Institute, Brisbane, Queensland 4006, Australia. ${ }^{2}$ Department of Epidemiology and Cancer Control, St. Jude Children's Research Hospital, Memphis, Tennessee 38105, USA. ${ }^{3}$ deCODE Genetics/Amgen, 101 Reykjavik, Iceland. ${ }^{4}$ Department of Biostatistics, University of Liverpool, Liverpool L69 3GL, UK. ${ }^{5}$ Wellcome Trust Centre for Human Genetics, University of Oxford, Oxford OX3 7BN, UK. ${ }^{6}$ KULeuven, Department of Development and Regeneration, Organ systems, 3000 Leuven, Belgium. ${ }^{7}$ Department of Obstetrics and Gynaecology, Leuven University Fertility Centre, University Hospital Leuven, 3000 Leuven, Belgium. ${ }^{8}$ Harvard T.H. Chan School of Public Health, Boston, Massachusetts 02115, USA. ${ }^{9}$ Channing Division of Network Medicine, Department of Medicine, Brigham and Women's Hospital and Harvard Medical School, Boston, Massachusetts 02115, USA. ${ }^{10}$ Division of Preventive Medicine, Brigham and Women's Hospital, Boston, Massachusetts 02215, USA. ${ }^{11}$ Institute for Molecular Bioscience, The University of Queensland, Brisbane, Queensland 4072, Australia. ${ }^{12}$ Institute of Medicine and Public Health, Vanderbilt University Medical Center, Nashville, Tennessee 37203, USA. ${ }^{13}$ Vanderbilt Genetics Institute, Division of Epidemiology, Institute of Medicine and Public Health, Department of Medicine, Vanderbilt University Medical Center, Nashville, Tennessee 37203, USA. ${ }^{14}$ Cognitive Science Department, University of California, San Diego, La Jolla, California 92093, USA. ${ }^{15}$ Institute of Biological Psychiatry, Mental Health Centre Sct. Hans, Copenhagen University Hospital, DK-2100 Copenhagen, Denmark. ${ }^{16}$ Endometriosis CaRe Centre, Nuffield Dept of Obstetrics \& Gynaecology, University of Oxford, John Radcliffe Hospital, Oxford OX3 9DU, UK. ${ }^{17}$ Department of Obstetrics and Gynecology, Niigata University Graduate School of Medical and Dental Sciences, Niigata 950-2181, Japan. ${ }^{18}$ Center for Integrative Medical Sciences, RIKEN, Yokohama 230-0045, Japan. ${ }^{19}$ Institute of Medical Sciences, The University of Tokyo, Tokyo 108-8639, Japan. ${ }^{20}$ Department of Obstetrics and Gynecology, Landspitali University Hospital, 101 Reykjavik, Iceland. ${ }^{21}$ Faculty of Medicine, School of Health Sciences, University of Iceland, 101 Reykjavik, Iceland. ${ }^{22}$ Vanderbilt Genetics Institute, Vanderbilt Epidemiology Center, Institute of Medicine and Public Health, Department of Obstetrics and Gynecology, Vanderbilt University Medical Center, Nashville, Tennessee 37203, USA. ${ }^{23}$ Department of Biomedicine, Aarhus University, DK8000 Aarhus, Denmark. ${ }^{24}$ iPSYCH, The Lundbeck Foundation Initiative for Integrative Psychiatric Research, DK-2100 Copenhagen, Denmark. ${ }^{25}$ Global Medical Affairs Fertility, Research and Development, Merck KGaA, Darmstadt, Germany. ${ }^{26} 23$ andMe, Inc., 899 W. Evelyn Avenue, Mountain View, California 94041, USA. ${ }^{27}$ Institute of Health and Biomedical Innovation, Queensland University of Technology, Queensland 4059, Australia. ${ }^{\star}$ These authors contributed equally to this work. Correspondence and requests for materials should be addressed to Y.S. (email: Yadav.Sapkota@stjude.org.) or to G.W.M. (email: g.montgomery1@uq.edu.au) or to D.R.N. (email: d.nyholt@qut.edu.au).

$\uparrow$ A full list of consortium members appears at the end of the paper
} 
E ndometriosis is a common gynecological disorder that affects $6-10 \%$ of women of reproductive age ${ }^{1}$ and $20-50 \%$ of women with infertility ${ }^{2}$. The disease is associated with pelvic pain and is primarily characterized by the presence of endometrium-like tissue outside the uterus. The etiology of endometriosis is complex, involving multiple genetic and environmental risk factors. The condition has an estimated total heritability of $0.47-0.51$ based on twin studies ${ }^{1,3}$, and a common SNP-based heritability of 0.26 (ref. 4).

Genome-wide association (GWA) studies have identified 11 independent single-nucleotide polymorphisms (SNPs) for endometriosis. These SNPs include rs10965235 in CDKN2BAS on chromosome 9p21.3 identified in a Japanese ancestry GWA study $^{5}$; rs1519761 on 2q23.3 identified in a US GWA study of European-ancestry women ${ }^{6}$; seven loci (rs7521902 near WNT4 on 1p36.12, rs13391619 in GREB1 on 2p25.1, rs4141819 on 2p14, rs7739264 near ID4 on 6p22.3, rs12700667 on 7p15.2, rs1537377 near CDKN2B-AS1 (independent of rs10965235) on 9p21.3 and rs10859871 near VEZT on 12q22) identified in a Europeanancestry GWA study ${ }^{7}$ and from a meta-analysis of European and Japanese ancestry GWA data ${ }^{8}$; and most recently rs17773813 near KDR on 4q12 and rs519664 in TTC39B on 9p22 in an Icelandic GWA study ${ }^{9}$. We also recently confirmed the suggested association of the IL1A gene locus on $2 \mathrm{q} 13$, by identifying genome-wide significant association between rs6542095 and endometriosis $^{10,11}$. Thus, bringing the total to 12 independent SNPs associated with endometriosis at the genome-wide significance level, of which all but one (rs10965235 in CDKN2BAS on 9p21.3, identified in the Japanese GWA study ${ }^{5}$ ) are polymorphic in populations of European ancestry. Of the 11 European SNP risk loci, eight SNPs have been replicated and robustly implicated as susceptibility loci for endometriosis ${ }^{10,12}$, the exceptions being rs1519761 (2q23.3), rs17773813 (4q12) and rs519664 (TTC39B) that are yet to be examined in an independent study.

To gain a better understanding of the genetic architecture of endometriosis, we sought to substantially expand upon the existing GWA data for endometriosis. Including 11 individual case-control data sets of European and Japanese ancestries (ten imputed using a recent 1000 Genomes Project reference panel and one directly genotyped), the current meta-analysis represents an approximate five-fold increase in the effective sample size ${ }^{13}$ in comparison to the previously largest multi-ethnic GWA metaanalysis of 4,604 endometriosis cases and 9,393 controls from Australia, United Kingdom and Japan ${ }^{8}$. In addition to replicating 9 of the 11 previously reported European risk loci, this GWA meta-analysis identified 5 novel loci significantly associated with endometriosis risk $\left(P<5 \times 10^{-8}\right)$, implicating genes involved in sex steroid hormone pathways (FN1, CCDC170, ESR1, SYNE1 and $F S H B$ ). Conditional analyses identified five novel secondary association signals at these implicated loci, including two at the ESR1 locus, resulting in a total of 19 independent SNPs robustly associated with endometriosis, which together explain up to $5.19 \%$ of variance in endometriosis.

\section{Results}

Study overview. This meta-analysis combined GWA data from 11 individual GWA case-control data sets, totalling 17,045 endometriosis cases and 191,858 controls of European and Japanese ancestries from Australia, Iceland, Belgium, the UK, the USA, Denmark and Japan. Individuals in the study are predominantly Europeans representing 93\% of the total effective sample size (cases and controls), with the remaining $\sim 7 \%$ being of Japanese descent. A brief summary of the 11 individual GWA case-control data sets is provided in Supplementary Data 1. Seven
GWA data sets (QIMRHCS, OX, BBJ, deCODE, Adachi-6 and Adachi-500 K) have been published previously $5,8-10,14,15$ and the remaining four are unpublished. Because individuals in the Adachi data set were genotyped using two different platforms (Affymetrix $500 \mathrm{~K}$ and 6.0), they were processed and analysed as separate GWA data sets. Details of individual data sets are provided in the Supplementary Information. All cases in the QIMRHCS, OX, deCODE and LEUVEN studies have surgically confirmed endometriosis and disease stage from surgical records using the revised American Fertility Society (rAFS) classification system $^{16}$; women were grouped into Grade A (rAFS I or II disease or some ovarian disease with a few adhesions), Grade B (rAFS III or IV disease) or unknown stage as described previously ${ }^{7}$. Diagnosis of endometriosis in other studies is based on self-reports or a combination of surgical records and self-report (see Supplementary Information for more details).

Each GWA case-control data set followed similar quality control procedures and was imputed separately using the same 1000 Genomes Project reference panel (March 2012 Release), with the exception being the 23andMe and deCODE studies that were imputed using the 1000 Genomes Project October 2010 haplotypes and whole genome sequence data $(\sim 30$ million sequence variants) of 8,453 Icelanders, respectively. The Adachi-6 data set consisted of only observed genotype data because individual-level genotypes were not available to carry out imputation. Genotypes (observed or imputed) in individual GWA case-control data sets were analysed and processed using similar approaches (see Methods section).

Primary GWA meta-analysis of all 17,045 endometriosis cases versus 191,858 controls, for the $6,979,035$ SNPs that passed quality control in at least $50 \%$ (6 or more) of the participating studies, was performed using a fixed-effect model. SNPs with $P<0.05$ in the primary GWA meta-analysis were further analysed after excluding cases with minimal or mild (Grade A) endometriosis (rAFS I or II disease) from the QIMRHCS, deCODE, LEUVEN and OX cohorts. A total of 391 SNPs reached conventional genome-wide significance $\left(P<5 \times 10^{-8}\right)$ and their association summary results are provided in Supplementary Data 2. SNPs showing suggestive association in the fixed-effect model and with evidence of heterogeneity $\left(P_{\text {het }}<0.05\right)$ were also analysed using the Han Eskin random-effects model (RE2) ${ }^{17}$. The $\mathrm{RE} 2$ model is similar to the traditional RE approach except it relaxes the conservative assumption in hypothesis testing and assumes no heterogeneity under the null hypothesis of no association. As such, it offers greater power under heterogeneity as compared with the conventional random-effects model. The genomic inflation factor $(\lambda)$ for the GWA meta-analysis was 1.12. Quantile-quantile (Q-Q) plots for the fixed-effect GWA meta-analysis and 11 individual GWA case-control data sets are provided in Supplementary Figs 1-12.

Risk loci associated with endometriosis. Of the 11 previously reported European SNP risk loci, nine reached genome-wide significance $\left(P<5 \times 10^{-8}\right)$ in the present study (Fig. 1 and Table 1); we did not confirm associations at 2 q23.3 and 9 p22. The Q-Q plot for the fixed-effect GWA meta-analysis including all endometriosis cases ('All') after excluding the nine loci (the most significant ('index') SNPs and $1 \mathrm{Mb}$ flanking region) is provided in Supplementary Fig. 13. SNP associations at loci on 1p36.12, 7 p15.2 and 9p21.3 remained genome-wide significant when secondary meta-analysis ('Grade B') was performed after excluding cases with known minimal or mild (Grade A) endometriosis (rAFS I or II disease) (Table 1). Furthermore, all nine previously reported loci produced larger effects (odds ratios (ORs)) in Grade $\mathrm{B}$ analysis compared to the analysis of all cases-an observation 


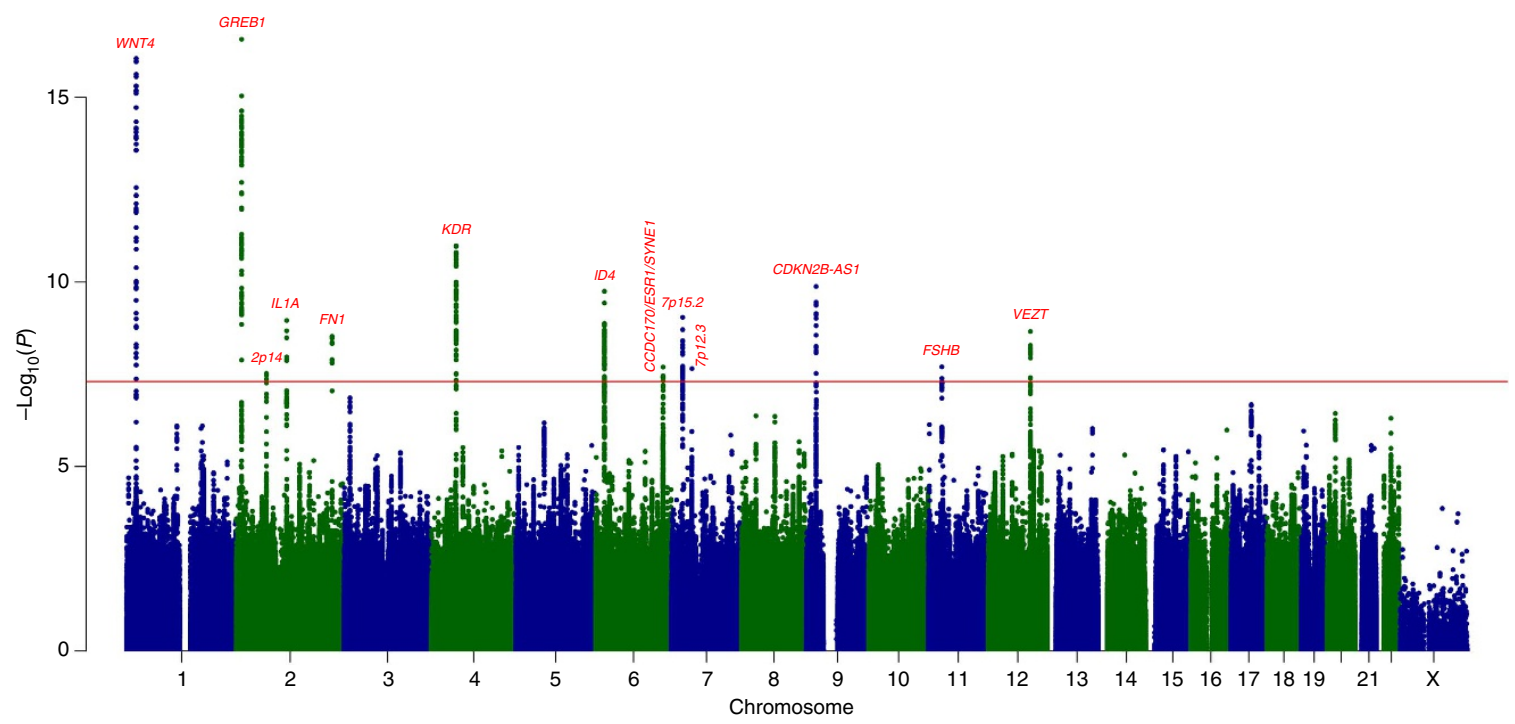

Figure 1 | Manhattan plot for genome-wide associations with endometriosis. Data are based on GWA meta-analysis of all endometriosis cases. The horizontal axis shows the chromosomal position, and the vertical axis shows the significance of tested markers combined in a fixed-effects meta-analysis. Markers that reached genome-wide significance $\left(P<5 \times 10^{-8}\right)$ are highlighted.

Table 1 | Summary of the GWA meta-analysis results for 14 genome-wide significant loci.

\begin{tabular}{|c|c|c|c|c|c|c|c|c|c|c|c|}
\hline \multirow[t]{2}{*}{ Chr } & \multirow[t]{2}{*}{ SNP } & \multirow[t]{2}{*}{ Position (bp) } & \multirow[t]{2}{*}{ RA } & \multirow[t]{2}{*}{ OA } & \multicolumn{4}{|c|}{ Meta-analysis (All) } & \multicolumn{2}{|c|}{ Meta-analysis (Grade B) } & \multirow{2}{*}{$\begin{array}{l}\text { Associated } \\
\text { gene/cytoband }\end{array}$} \\
\hline & & & & & $\mathbf{R A F}_{\text {EUR }}$ & RAF JPT $_{\text {JP }}$ & OR $(95 \% \mathrm{CI})$ & $P$ value & OR $(95 \% \mathrm{CI})$ & $P$ value & \\
\hline \multicolumn{12}{|c|}{ Previously reported loci } \\
\hline 2 & rs11674184 & 11721535 & $\mathrm{~T}$ & $\mathrm{G}$ & 0.61 & 0.54 & $1.13(1.10-1.15)$ & $2.67 \times 10^{-17}$ & $1.18(1.10-1.24)$ & $1.94 \times 10^{-6}$ & GREB1/2p25.1 \\
\hline 2 & rs6546324 & 67856490 & $A$ & $\mathrm{C}$ & 0.31 & 0.21 & $1.08(1.05-1.11)$ & $3.01 \times 10^{-8}$ & $1.19(1.11-1.26)$ & $3.71 \times 10^{-7}$ & ETAA1/2p14 \\
\hline 2 & rs10167914 & 113563361 & G & A & 0.30 & 0.75 & $1.12(1.08-1.15)$ & $1.10 \times 10^{-9}$ & $1.15(1.07-1.21)$ & $7.59 \times 10^{-5}$ & IL1A/2q13 \\
\hline 7 & rs12700667 & 25901639 & $A$ & G & 0.74 & 0.20 & $1.10(1.07-1.13)$ & $9.08 \times 10^{-10}$ & $1.28(1.19-1.36)$ & $6.69 \times 10^{-11}$ & $7 p 15.2$ \\
\hline 9 & rs1537377 & 22169700 & C & $\mathrm{T}$ & 0.40 & 0.39 & $1.09(1.06-1.12)$ & $1.33 \times 10^{-10}$ & $1.21(1.13-1.27)$ & $6.31 \times 10^{-9}$ & CDKN2B-AS1/9p21.3 \\
\hline 12 & rs4762326 & 95668951 & $\mathrm{~T}$ & $\mathrm{C}$ & 0.47 & 0.48 & $1.08(1.05-1.11)$ & $2.20 \times 10^{-9}$ & $1.15(1.08-1.21)$ & $1.08 \times 10^{-5}$ & VEZT/12q22 \\
\hline \multicolumn{12}{|c|}{ Novel loci } \\
\hline 2 & rs1250241 & 216295312 & $\mathrm{~T}$ & A & 0.29 & 0.06 & $1.06(1.03-1.09)$ & $6.20 \times 10^{-5}$ & $1.23(1.15-1.30)$ & $2.99 \times 10^{-9}$ & $F N 1 / 2 q 35$ \\
\hline 6 & rs1971256 & 151816011 & C & $\mathrm{T}$ & 0.20 & 0.35 & $1.09(1.06-1.13)$ & $3.74 \times 10^{-8}$ & $1.28(1.19-1.36)$ & $1.50 \times 10^{-10}$ & $C C D C 170 / 6 q 25.1$ \\
\hline
\end{tabular}

Chr, Chromosome; SNP, single-nucleotide polymorphism; Genomic position is shown relative to GRCh37 (hg19); GWA, genome-wide association; RA, risk allele; OA, other allele; OR, odds ratio with respect to $\mathrm{RA}$; $\mathrm{Cl}$, confidence interval; $\mathrm{RAF}_{\mathrm{EUR}}$, average risk allele frequency in European samples; $\mathrm{RAF}_{\mathrm{JPT}}$, average risk allele frequency in Japanese samples.

consistent with previous reports of greater genetic loading in moderate-to-severe endometriosis. Regional association plots of the nine previously reported loci based on the fixed-effect metaanalysis results including all and Grade B endometriosis cases are provided in Supplementary Figs 14-22.

In addition to robustly implicating nine previously identified endometriosis SNP loci, the meta-analysis identified five new genomic regions harbouring risk loci for endometriosis (Fig. 1 and Table 1). In the 'All' fixed-effect meta-analysis, we observed genome-wide significant evidence for risk loci in CCDC170 on 6q25.1 (rs1971256: OR (95\% confidence interval $(\mathrm{CI}))=1.09$ $\left.(1.06-1.13) ; P_{\text {all }}=3.74 \times 10^{-8}\right)$, in SYNE1 on 6 q25.1 (rs71575922: OR $\left.(95 \% \mathrm{CI})=1.11(1.07-1.15) ; P_{\text {all }}=2.02 \times 10^{-8}\right)$ and near FSHB on 11p14.1 (rs74485684: OR $(95 \% \mathrm{CI})=1.11(1.07-1.15)$; $\left.P_{\text {all }}=2.00 \times 10^{-8}\right)$. The 'Grade B' fixed-effect meta-analysis implicated rs 1250241 in FN1 on $2 \mathrm{q} 35$ (OR $(95 \% \mathrm{CI})=1.23$ $\left.(1.15-1.30) ; P_{\text {Grade B }}=2.99 \times 10^{-9}\right)$ and rs74491657 on $7 \mathrm{p} 12.3$
$\left(\mathrm{OR}(95 \% \mathrm{CI})=1.46(1.28-1.59) ; P_{\text {Grade } \mathrm{B}}=2.24 \times 10^{-8}\right)$ as genome-wide significantly associated with endometriosis risk (Table 1). Regional association plots of loci on 6q25.1 (CCDC170 and SYNE1) and 11p14.1 (FSHB) based on fixed-effect metaanalysis results including all cases, and of loci on $2 \mathrm{q} 35$ and $7 \mathrm{p} 12.3$ based on results from fixed-effect meta-analysis for Grade B cases are provided in Fig. 2. Associations at the 6q25.1 CCDC170 and SYNE1 loci remained genome-wide significant in the 'Grade B' analysis, with larger effects (ORs) for the risk allele in comparison to those based on analysis of all endometriosis cases (Table 1). Regional association plots of the five newly identified loci based on fixed-effect meta-analysis including all and Grade B endometriosis cases are provided in Fig. 2 and Supplementary Fig. 23. Forest plots of risk allele effects (ORs) for the index SNPs at 14 loci in the individual GWA case-control data sets, and for the 'All' and 'Grade B' fixed-effect meta-analyses are given in Fig. 3 and Supplementary Figs 24-33. 
a

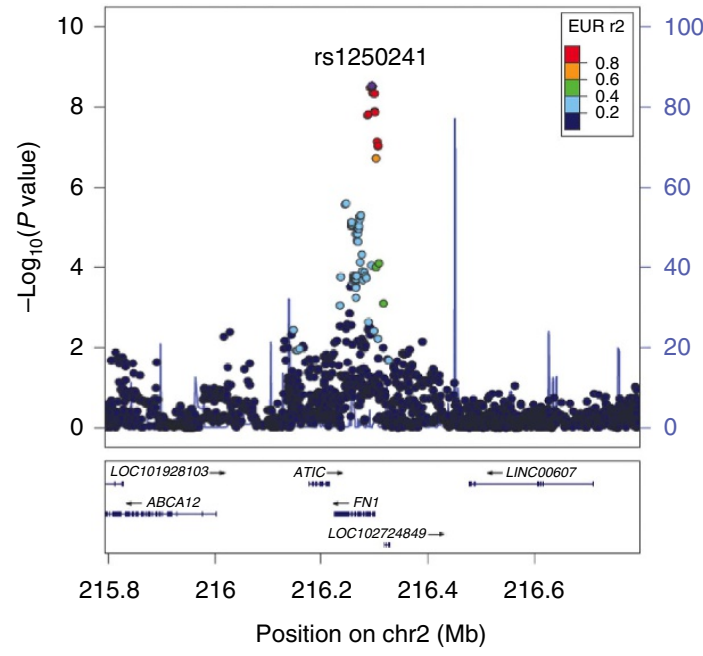

C

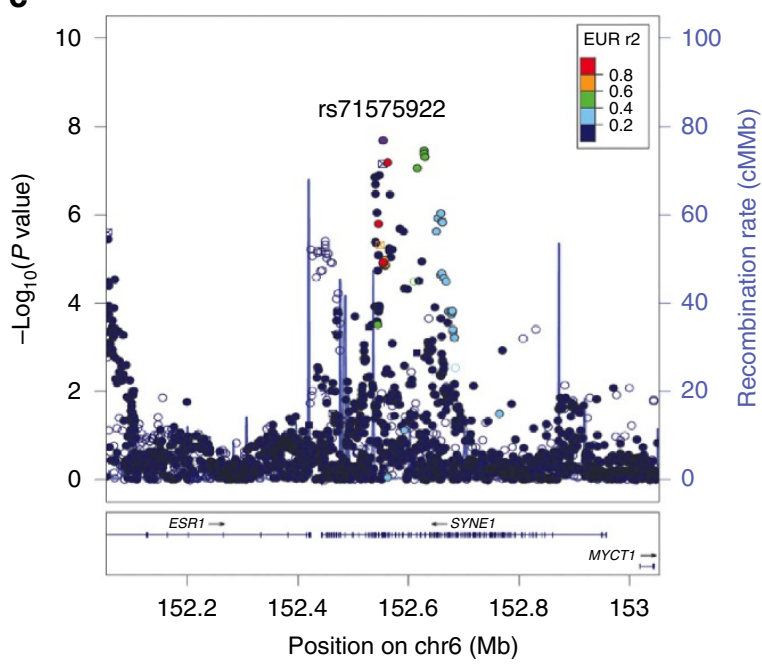

e

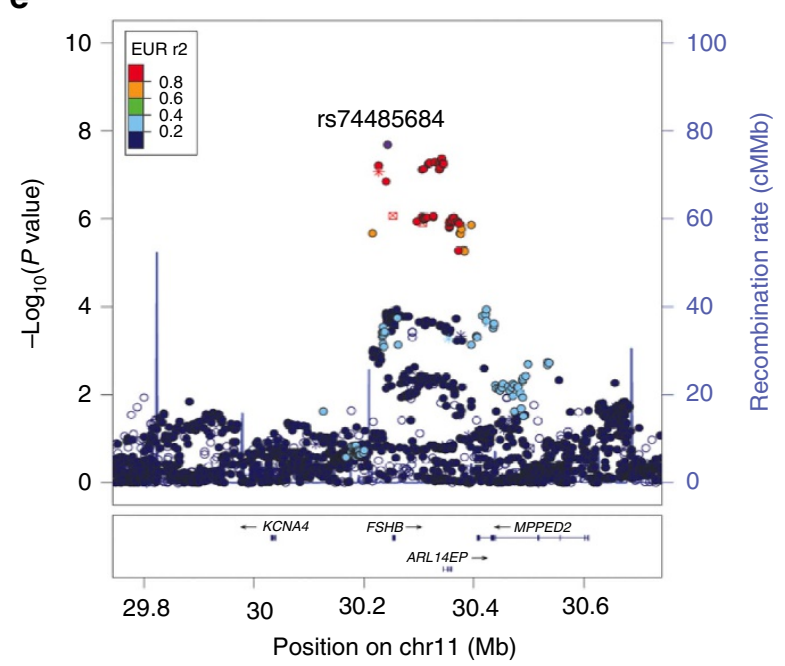

b

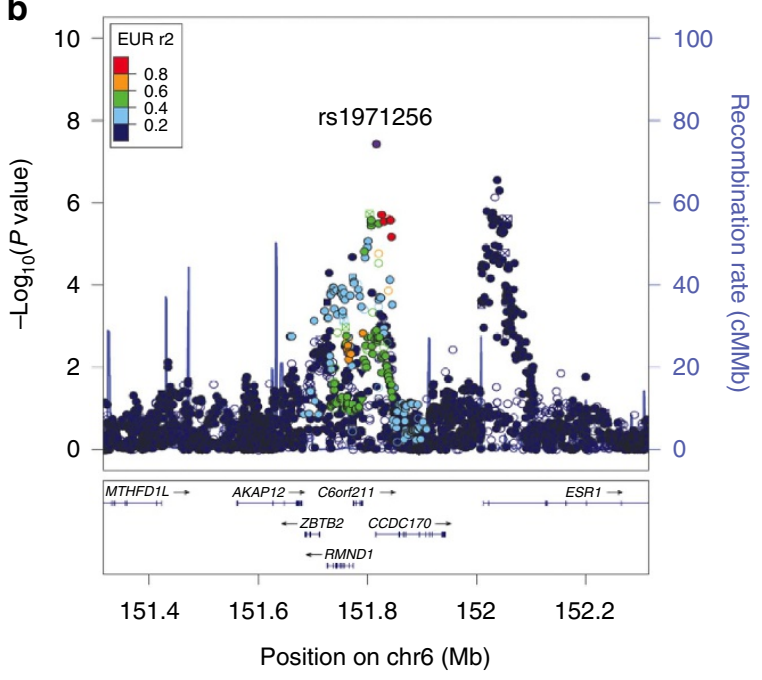

d

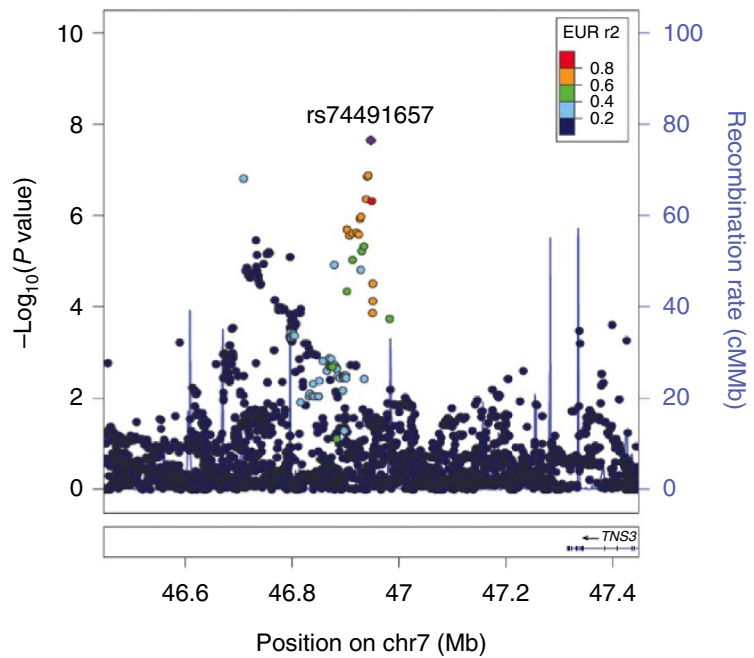

Figure 2 | LocusZoom plots of five genome-wide significant endometriosis loci. Association with endometriosis is expressed as $-\log _{10}(P$ value $)$ for five new genome-wide significant loci: FN1 2q35 (2a), CCDC170 on 6q25.1 (2b), SYNE1 on 6q25.1 (2c), 7p12.3 (2d), and near FSHB on 11p14.1 (2e). Results for $2 q 35$ and $7 p 12.3$ are based on analysis including only moderate-to-severe ('Grade B') endometriosis cases. SNPs are shown as circles, diamonds or squares (filled or unfilled), with the top SNP represented by purple colour. All other SNPs are colour coded according to the strength of LD with the top SNP (as measured by $r^{2}$ in the European 1000 Genomes data). 
a

QIMRHCS

deCODE

LEUVEN

OX

GWA meta-analysis (grade B)

C

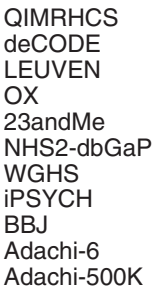

Adachi-500K

GWA meta-analysis (all)

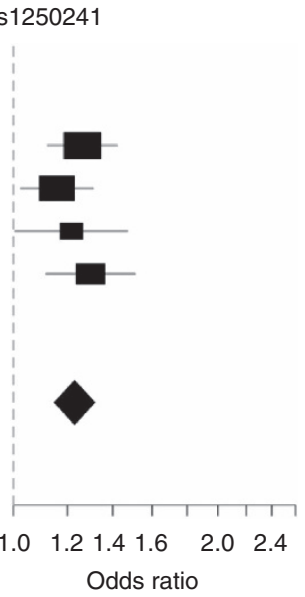

rs1971256

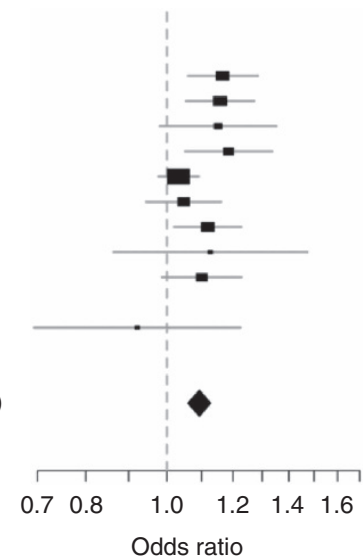

e

QIMRHCS

deCODE

LEUVEN

OX

23andMe

NHS2-dbGaP

WGHS

PSYCH

BBJ

Adachi-6

Adachi-500K

GWA meta-analysis (all)

rs74485684

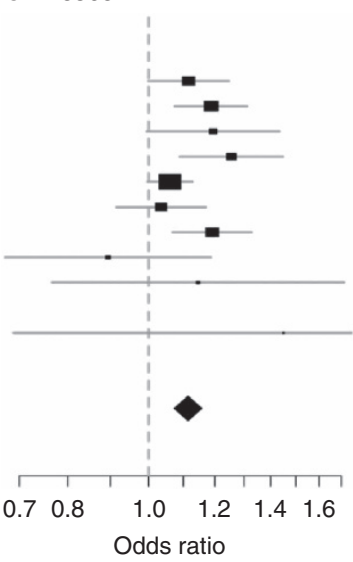

b

rs71575922

QIMRHCS

deCODE

LEUVEN

OX

23andMe

NHS2-dbGaP

WGHS

iPSYCH

BBJ

Adachi-6

Adachi-500K

GWA meta-analysis (all)

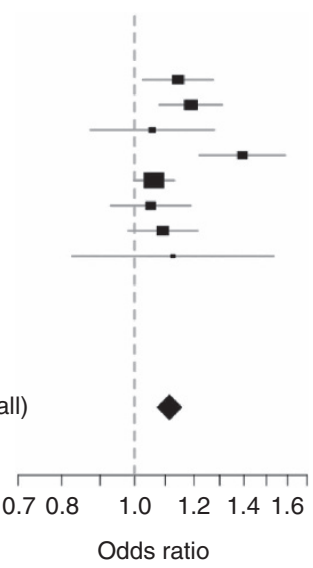

d

rs74491657

QIMRHCS

deCODE

LEUVEN

OX

GWA meta-analysis

(grade B)

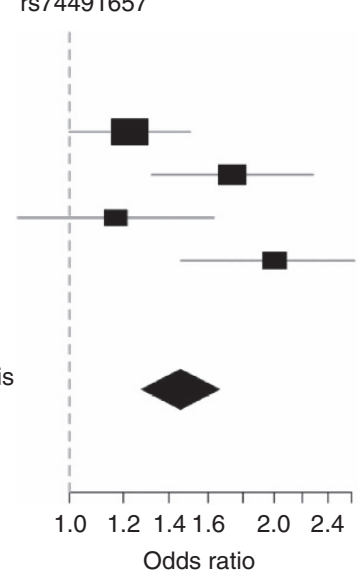

Figure 3 | Forest plots showing risk allele effects for five endometriosis loci. Risk allele effects for the five new genome-wide significant loci in the individual case-control data sets and GWA meta-analysis: FN1 2q35 (3a), CCDC170 on 6q25.1 (3b), SYNE1 on 6q25.1 (3c), 7p12.3 (3d), and near FSHB on 11 p14.1 (3e). Results for 2q35 and 7p12.3 are based on analysis including only moderate-to-severe ('Grade B') endometriosis cases. Risk allele effects of the remaining three SNPs are from analysis including all endometriosis cases.

Distinct association signals at endometriosis risk loci. To identify distinct secondary association signals at the 14 loci, we used the genome-wide complex trait analysis (GCTA) software ${ }^{18}$ to perform approximate conditional analysis based on summary statistics from meta-analysis including all endometriosis cases. For the FN1 2q35 and 7p12.3 loci, we used summary statistics from meta-analysis including Grade B endometriosis cases. We conservatively defined a locus as the chromosomal region $500 \mathrm{~kb}$ up- and down-stream of the index SNP at the locus.
We estimated the effective number of independent SNPs to be 11,631 across all 14 regions. We therefore used a region-wide Bonferroni adjusted significant threshold of $P<4.3 \times 10^{-6}$ to declare a secondary association signal if a SNP achieved this threshold after conditioning on the index SNP at each locus. GCTA identified five secondary signals including one (rs77294520 near GREB1) on 2p25.1; two (rs2206949 in ESR1 and rs17803970 in SYNE1) on 6q25.1, and two (rs10757272 in CDKN2B-AS1 and rs1448792) on 9p21.3 (Table 2). Of these, 
rs77294520 on 2p25.1 also remained significant in Grade B analyses with a larger effect (OR). Results for all SNPs with $P<4.3 \times 10^{-6}$ in the GCTA conditional analysis based on summary statistics including all studies are provided in Supplementary Data 3. We also performed additional conditional analysis using European samples alone. Except for near region-wide significance for rs17803970 on 6q25.1 (SYNE1; $\left.P=4.59 \times 10^{-6}\right)$ and for rs10757272 on $9 \mathrm{p} 21.3$ (CDKN2B-AS1; $\left.P=1.40 \times 10^{-5}\right)$, the remaining three secondary association signals persisted with region-wide significance when analysis was restricted to Europeans studies only (Supplementary Data 4). Furthermore, there was no linkage disequilibrium (LD) or very low LD $\left(r^{2}<0.03\right)$ between the index SNPs and the five secondary association signals on 2p25.1, 6q25.1 and 9p21.3 in both European and Japanese populations (Supplementary Data 5), suggesting that the results are not influenced by differences in LD patterns across European and Japanese populations. Regional association and forest plots for the five secondary signals are provided in Supplementary Figs 34-42. Taken together, these data implicate 19 independent SNPs at 14 distinct genomic loci, including four on $6 \mathrm{q} 25.1-\mathrm{a}$ locus containing a cluster of genes including ESR1.

On the basis of the fixed-effect GWA meta-analysis results including all endometriosis cases from European studies, the nine previously reported SNPs explain $0.97 \%$ of the phenotypic variance on the liability scale ${ }^{19}$. The 10 new SNPs identified in this study together explain a further $0.78 \%$, totalling to $1.75 \%$ of the phenotypic variance explained for endometriosis. More importantly, the 19 independent SNPs together explained 5.19\% of the phenotypic variance in Grade B endometriosis cases, of which $2.46 \%$ was explained by the 10 new SNPs.

SNP effects based on ancestry and endometriosis definition. Of the 14 distinct loci, 6 (2p14, 2q35, 4q12, 6q25.1 (SYNE1), $7 \mathrm{p} 15.2$ and 12q22) showed evidence of between-study heterogeneity $\left(P_{\text {het }}<0.05 ; I^{2}: 13.30-20.33\right)$ in fixed-effect meta-analysis including all endometriosis cases; however, after appropriately modelling the observed heterogeneity in the RE2 model, these associations remained genome-wide significant (Supplementary Data 2).

None of the six loci showed between-study heterogeneity in analyses restricted to Japanese alone and self-reported endometriosis cases (Supplementary Data 6). In Europeans, five loci (2p14, 2q35, 4q12, 6q25.1 (SYNE1) and 7p15.2) showed heterogeneity in allelic associations, and except for $6 \mathrm{q} 25.1$ (SYNE1), this trend persisted in surgically confirmed endometriosis cases. However, except for $2 \mathrm{p} 14$ and $4 \mathrm{q} 12$, this heterogeneity attenuated in moderate-to-severe endometriosis cases (Supplementary Data 2). All five loci except 2p14 produced larger effect sizes for surgically confirmed endometriosis than diagnosis based on self-reports. With respect to $2 \mathrm{p} 14$, residual betweenstudy heterogeneity may be driven by opposite direction of effect in two out of the eight European studies (Supplementary Data 2 and Supplementary Fig. 26).

Most SNPs showed larger effect sizes in Japanese populations in comparison with results from Europeans alone (Supplementary Data 6). Whereas all 19 SNPs showed significant associations with endometriosis $\left(P<2.6 \times 10^{-3}\right.$ after multiple testing) in Europeans, only six SNPs showed significance in Japanese alone, although importantly, direction of effects for all SNPs were concordant with those produced in the meta-analysis. For surgically confirmed endometriosis, all but two SNPs (rs2206949 on 6q25.1 and rs10757272 on 9p21.3) showed significant association with endometriosis $\left(P<2.6 \times 10^{-3}\right.$ after multiple testing).
Endometriosis risk SNPs associated with other traits. We checked genome-wide significant associations of the 19 SNPs with other diseases or traits using the NHGRI GWAS catalogue ${ }^{20}$. We searched for 395 SNPs including the 19 SNPs as well as all other SNPs in high LD $\left(r^{2}>0.7\right)$ with the 19 SNPs (Supplementary Data 7). Of these, we observed associations with multiple diseases or traits, including epithelial ovarian cancer ${ }^{21}$, low-density lipoprotein cholesterol ${ }^{22}$, coronary heart disease ${ }^{23}$, luteinizing hormone and follicle-stimulating hormone levels, and age at onset for menopause ${ }^{24}$.

We did not observe statistically significant genetic correlation between endometriosis and 199 common complex traits, based on $\mathrm{LD}$ score regression analysis ${ }^{25}$ using $\mathrm{LD}$ hub ${ }^{26}$. Genetic correlations with nominal $P<0.05$ are provided in Supplementary Figs 43,44.

Considering that SNPs at $6 \mathrm{q} 25.1$ are reported to be associated with breast cancer and related phenotypes, we investigated for overlap of association signals between breast cancer and endometriosis. A recent study based on the custom-designed iCOGS data in 118,816 women reported evidence for at least 5 independent risk variants at $6 \mathrm{q} 25.1$, each associated with different breast cancer phenotypes, including oestrogen receptor, human ERBB2 tumour subtypes, mammographic density, and tumour grade $^{27}$. We found no overlap $\left(\mathrm{LD} r^{2}<0.17\right)$ between these five breast cancer signals and our four independent SNPs at the 6q25.1 (ESR1) locus. We therefore obtained association summary results of 101 SNPs with genome-wide significance for overall breast cancer from Dunning et al. ${ }^{27}$, and cross-checked with our GWA meta-analysis results (Supplementary Data 9). Of these, 23 SNPs also showed associations $(P<0.05)$ with endometriosis. The risk allele of six SNPs was the same for both breast cancer and endometriosis, including four SNPs (rs851981, rs851980, rs2206948 and rs150182883) that are in strong $\operatorname{LD}\left(r^{2} \geq 0.60\right)$ with our secondary association signal rs2206949 at 6q25.1 (ESR1). The secondary endometriosis risk SNP rs2206949 was also strongly associated with overall breast cancer $\left(P=5.5 \times 10^{-6}\right)$. Based on the 1000 Genomes Project European reference data, the 101 and 23 SNP-sets correspond to four and two independent SNPs, respectively, thereby suggesting significant genetic overlap between overall breast cancer and endometriosis $(P=0.02$; binomial test) at the 6q25.1 (ESR1) locus.

Genes associated with endometriosis. A genome-wide gene-based test using VEGAS2 (ref. 28) identified 18 genes that reached our conservative gene-based threshold of $P<2.23 \times 10^{-6}$ (Supplementary Data 10); we also provide results for all genes with combined $P<0.05$ for reference. Of these, 12 genes are located at six GWA SNP risk loci including 1 p36.12 (WNT4, LINC00339, LOC101928043 and CDC42), 2p25.1 (GREB1), 2q13 (IL1A and CKAP2L), 7p15.2 (RNU6-16P), 9p21.3 (CDKN2A) and 12q22 (MIR331, MIR3685 and VEZT). Notably, the remaining six gene-based genome-wide significant association signals were at three novel genomic regions 1q24.3 (DNM3OS, MIR214 and MIR3120), 9q22.32 (MIR23B, MIR27B) and 16p13.3 (LINC00921).

Fine-mapping of endometriosis risk loci. To identify potential causal variants responsible for the 19 independent association signals, we performed fine-mapping analysis based on our GWA meta-analysis results including all studies except for the Adachi-6 data set, as well as using results from only Europeans. (Supplementary Data 11). We assumed a single causal variant for each association signal and constructed a 99\% credible set of variants including SNPs within $500 \mathrm{~kb}$ of the index SNP. Except for the 6q25.1 (SYNE1) locus, the length of the 99\% credible 


\begin{tabular}{|c|c|c|c|c|c|c|c|c|c|c|c|c|c|c|}
\hline Chr & SNP & $\begin{array}{c}\text { Position } \\
\text { (bp) }\end{array}$ & RA & OA & $\mathrm{RAF}_{\mathrm{EUR}}$ & RAF JPT $_{\text {JP }}$ & $\begin{array}{l}\text { Endometriosis } \\
\text { cases }\end{array}$ & RAF & OR $(95 \% \mathrm{Cl})$ & $P$ value & Freq_ref & $\begin{array}{c}\mathrm{OR}_{\text {cond }} \\
(95 \% \mathrm{Cl})\end{array}$ & $P_{\text {cond }}$ & $\begin{array}{c}\text { Associated gene } \\
\text { cytoband }\end{array}$ \\
\hline 2 & rs77294520 & 11660955 & c & G & 0.147 & - & $\begin{array}{l}\text { All } \\
\text { Grade B }\end{array}$ & $\begin{array}{l}0.147 \\
0.150\end{array}$ & $\begin{array}{l}1.16(1.11-1.21) \\
1.29(1.18-1.42)\end{array}$ & $\begin{array}{l}9.91 \times 10^{-13} \\
1.45 \times 10^{-8}\end{array}$ & $\begin{array}{l}0.144 \\
0.147\end{array}$ & $\begin{array}{r}1.13(1.09-1.18) \\
1.26(1.15-1.37)\end{array}$ & $\begin{array}{l}1.67 \times 10^{-9} \\
4.76 \times 10^{-7}\end{array}$ & GREB1/2p25.1 \\
\hline 6 & rs2206949 & 152037556 & T & c & 0.270 & 0.162 & $\begin{array}{l}\text { All } \\
\text { Grade B }\end{array}$ & $\begin{array}{l}0.262 \\
0.275\end{array}$ & $\begin{array}{l}1.10(1.06-1.14) \\
1.09(1.01-1.17)\end{array}$ & $\begin{array}{l}2.73 \times 10^{-7} \\
2.49 \times 10^{-2}\end{array}$ & $\begin{array}{l}0.284 \\
0.286\end{array}$ & $\begin{array}{l}1.11(1.07-1.15) \\
1.11(1.03-1.19)\end{array}$ & $\begin{array}{l}2.68 \times 10^{-8} \\
5.19 \times 10^{-3}\end{array}$ & ESR1/6q25.1 \\
\hline 6 & rs17803970 & 152553718 & A & T & 0.918 & 0.970 & $\begin{array}{l}\text { All } \\
\text { Grade B }\end{array}$ & $\begin{array}{l}0.920 \\
0.921\end{array}$ & $\begin{array}{l}1.15(1.09-1.21) \\
1.35(1.18-1.53)\end{array}$ & $\begin{array}{l}7.04 \times 10^{-8} \\
4.75 \times 10^{-6}\end{array}$ & $\begin{array}{l}0.919 \\
0.918\end{array}$ & $\begin{array}{r}1.13(1.07-1.18) \\
1.27(1.12-1.44)\end{array}$ & $\begin{array}{l}3.25 \times 10^{-6} \\
2.21 \times 10^{-4}\end{array}$ & SYNE1/6q25.1 \\
\hline 9 & rs10757272 & 22088260 & c & T & 0.521 & 0.383 & $\begin{array}{l}\text { All } \\
\text { Grade B }\end{array}$ & $\begin{array}{l}0.504 \\
0.524\end{array}$ & $\begin{array}{l}1.07(1.04-1.10) \\
1.09(1.02-1.16)\end{array}$ & $\begin{array}{r}2.60 \times 10^{-7} \\
1.12 \times 10^{-2}\end{array}$ & $\begin{array}{l}0.506 \\
0.511\end{array}$ & $\begin{array}{l}1.07(1.04-1.10) \\
1.08(1.01-1.15)\end{array}$ & $\begin{array}{l}6.35 \times 10^{-7} \\
1.71 \times 10^{-2}\end{array}$ & $\begin{array}{c}\text { CDKN2B-AST/ } \\
9 p 21.3\end{array}$ \\
\hline 9 & rs1448792 & 22641633 & G & A & 0.757 & 0.661 & $\begin{array}{l}\text { All } \\
\text { Grade B }\end{array}$ & $\begin{array}{l}0.741 \\
0.759\end{array}$ & $\begin{array}{l}1.08(1.05-1.12) \\
1.06(0.98-1.14)\end{array}$ & $\begin{array}{l}1.79 \times 10^{-7} \\
1.21 \times 10^{-1}\end{array}$ & $\begin{array}{l}0.751 \\
0.753\end{array}$ & $\begin{array}{l}1.08(1.05-1.11) \\
1.05(0.97-1.13)\end{array}$ & $\begin{array}{l}7.03 \times 10^{-7} \\
1.99 \times 10^{-1}\end{array}$ & $9 p 21.3$ \\
\hline
\end{tabular}

Chr, Chromosome; SNP, single-nucleotide polymorphism; Genomic position is shown related to GRCh37 (hg19); Freq_ref, frequency of the risk allele in the reference sample; LD, linkage disequilibrium; $\mathrm{RA}$, risk allele; $\mathrm{OA}$, other allele; RAF, OR $(95 \% \mathrm{Cl})$ and $P$, risk allele frequency, odds ratio and $95 \%$ confidence interval, and $P$ value from the meta-analysis; OR $\mathrm{O}$ cond $(95 \% \mathrm{CI})$ and $P_{\text {cond, }}$ odds ratio and $95 \%$ confidence interval, and $P$ value from conditional analyses; $R_{\text {AF }}$ EUR, risk allele frequency in Europeans; $R A F_{J P T}$, risk allele frequency in Japanese;

interval and the number of credible SNPs for all association signals were smaller in analysis including all studies compared to the results restricted to only Europeans. Based on results from all studies, the smallest $99 \%$ credible interval was $\sim 16.52 \mathrm{~kb}$ observed for rs11674184 on $2 \mathrm{p} 25.1$ and the largest was $\sim 604.80 \mathrm{~kb}$ for the $6 \mathrm{q} 25.1$ (SYNE1). The $99 \%$ credible sets for the 19 independent associations using GWA meta-analysis results including all studies and only Europeans are provided in Supplementary Data 12.

Bioinformatic analyses of endometriosis risk loci. We then examined the cis associations between the 19 independent SNPs and other SNPs in high LD $\left(r^{2}>0.7\right)$ with the lead SNPs (Supplementary Data 7), and expression of nearby genes in whole blood, breast, cervix, muscle, ovary, uterus and adipose tissues using the GTEx eQTL portal ${ }^{29}$. Of these, the most relevant tissue for endometriosis is a small set of 32 uterine samples which are a mixture of both endometrium and myometrium. We found strong significant associations (false discovery rate $(\mathrm{FDR})<0.05$ ) between a SNP and expression of nearby genes in subcutaneous adipose tissues (Supplementary Data 13). Risk allele (G) of rs56376645 on 7p15.2 which is associated with endometriosis at genome-wide significance $(\mathrm{OR} \quad(95 \% \mathrm{CI})=1.09 \quad(1.06-1.12)$; $\left.P_{\text {all }}=1.93 \times 10^{-8}\right)$ also showed strong associations (beta $=0.38$; $\left.P=1.44 \times 10^{-7} ; \mathrm{FDR}=1.28 \times 10^{-3}\right)$ with increased expression of AC003090.1 (Supplementary Fig. 45).

To identify potentially causal genes underlying the identified endometriosis associations, we used a novel method ${ }^{30}$, summary data-based Mendelian randomization (SMR), which exploits the concept of Mendelian Randomisation (MR), to test for the causative effect of an exposure (that is, gene expression) on a phenotypic outcome (that is, endometriosis) using a genetic (SNP) variant as an instrumental variable. We used the method to identify causal genes at our endometriosis risk loci, using the GWA meta-analysis summary results from all studies including all endometriosis cases, and the eQTL summary data from Westra et al. ${ }^{31}$, an eQTL meta-analysis of 5,311 samples from peripheral blood, with SNPs imputed to the HapMap2 reference panel. The SMR analysis identified two potential causal genes, CDC42 (rs2268177; $\left.P_{\mathrm{SMR}}=1.07 \times 10^{-12}\right)$ and VEZT $(\mathrm{rs} 14121 ;$ $\left.P_{\mathrm{SMR}}=3.41 \times 10^{-6}\right)$, underlying endometriosis loci at $1 \mathrm{p} 36.12$ and 12q22, respectively (Supplementary Data 14). For rs2268177, there was no significant evidence of heterogeneity $\left(P_{\text {HEIDI }}=0.065\right)$ in effect sizes of dependent SNPs at this associated region and therefore it is very likely that rs2268177 contributes to both endometriosis risk and the expression level of $C D C 42$. Significant heterogeneity $\left(P_{\text {HEIDI }}<0.05\right)$ may indicate the possibility of two causal variants at a locus: one affecting endometriosis risk and the other affecting expression level of the gene (Supplementary Data 15). Significant heterogeneity $\left(P_{\text {HEIDI }}=0.004\right)$ for $\mathrm{rs} 14,121$ was observed, suggesting that the observed causal effect of VEZT expression on risk of endometriosis may be due to colocalization, but this needs to be investigated further.

DEPICT $^{32}$ analysis provided little support for potential genes, pathways, tissues or cell types reaching multiple testing threshold $(\mathrm{FDR} \leq 0.05)$. However, when using results with evidence of genome-wide suggestive association $\left(P<1 \times 10^{-5}\right)$ in either all or Grade B meta-analysis, DEPICT provided evidence for significant enrichment (FDR $\leq 0.05)$ of COPB1 PPI subnetwork gene set (Supplementary Data 16). Additionally, suggestive evidence for enrichment $(F D R \leq 0.2)$ was observed for ten tissues, including female genitalia, uterus, endocrine glands, endometrium, ovary and Fallopian tubes (Supplementary Data 16).

\section{Discussion}

We conducted a GWA meta-analysis of $\sim 7$ million SNPs in 17,045 endometriosis cases and 191,596 controls, confirmed 9 out of 11 previously reported European risk loci, and identified five new genomic regions in or near CCDC170, SYNE1, FSHB, FN1 and $7 \mathrm{p} 12.3$ harbouring endometriosis risk loci. This study represents a nearly fivefold increase in sample size in comparison with the previously largest endometriosis discovery GWA study and provided evidence for five secondary association signals including ESR1. The variance explained by the ten newly identified SNPs in all and Grade B cases was $0.78 \%$ and $2.46 \%$, bringing the total variance explained for endometriosis to $1.75 \%$ and $5.19 \%$, respectively, when considering all 19 associated SNPs. Importantly, our results highlight key genes involved in hormone metabolism that are likely to play a major role in endometriosis pathogenesis, thereby advancing current knowledge of endometriosis biology.

Previous GWA studies of endometriosis have implicated WNT signalling, oestrogen responsive genes and genes involved in the actin cytoskeleton and cellular adhesion ${ }^{12,33}$. Target genes in most regions are yet to be identified, but there is evidence to support candidates in several regions as previously described ${ }^{34}$. The most strongly associated (index) SNP for endometriosis at the WNT4 locus on chromosome 1p36.12 is also the index SNP for ovarian cancer ${ }^{35}$ and the risk mechanism likely acts through inverse regulation of CDC42 and LINC00339 (ref. 36). The index SNP associated with endometriosis on chromosome 2 p25.1 is a common splice variant in the oestrogen-responsive growth 
regulation by oestrogen in breast cancer 1 (GREB1) gene g $^{33,37}$ and SNPs associated with endometriosis on chromosome $12 \mathrm{q} 22$ increase expression of the transmembrane adherens junctions protein coding gene vezatin (VEZT) in RNA from blood and endometrium $^{38}$. We confirm association near the kinase insert domain receptor $(K D R)$ gene that was recently reported by the Icelandic GWA study 9 . The gene encodes vascular endothelial growth factor receptor 2, which promotes proliferation, survival, migration and differentiation of endothelial cells. $K D R$ is responsive to steroid hormones; expression of $K D R$ in endometrial stromal cells isolated from proliferative phase endometrium was significantly increased by oestrogen and medroxyprogesterone acetate in vitro ${ }^{39}$. During the premenstrual phase in both humans and macaques, KDR expression was significantly increased in the stromal cells of the endometrium ${ }^{40}$. Results from this meta-analysis support and extend these observations, but further functional studies are needed to identify and confirm the causal genes in all regions.

We identified several independent signals in a region that includes ESR1 encoding oestrogen receptor 1 on chromosome 6 25.1. Endometriosis is an oestrogen-dependent disease. Symptoms occur after puberty and oestrogen action contributes to pathological processes including growth of lesions and inflammation, and to the symptoms including pain ${ }^{41,42}$. Our primary meta-analysis identified two SNPs at this locus-rs1971256 in CCDC170 and rs71575922 in SYNE1-located up and downstream of ESR1, respectively. Conditional analysis identified a further two independent associations at this locus, including rs2206949 in ESR1 and rs17803970 in SYNE1. The 6q25.1 region is a well-established susceptibility locus for breast cancer in both Europeans and Asians ${ }^{27,43,44}$. Variants at this locus have recently been associated with overall breast cancer and its sub-phenotypes including tumour subtypes, mammographic density and tumour grade ${ }^{27}$. Of the four independent endometriosis SNPs at this locus, the association signal for rs2206949 in intron 2 of ESR1 overlaps with the signal observed for overall breast cancer. In fact, we observed a significant $(P=0.02$; binomial test) genetic overlap (sharing) between genetic risk for overall breast cancer and endometriosis at this locus, highlighting the importance of hormonal influences of both diseases which warrants further detailed exploration.

Our results support a role for a functional effect associated with endometriosis on chromosome $11 \mathrm{p} 14.1$. The association signal includes an LD region beginning upstream of FSHB and extending across to ARL14EP. The strongest signal was for SNP rs74485684 (risk allele $\mathrm{T}, \mathrm{RAF}=0.84, \mathrm{OR}=1.11$ ) located $10,276 \mathrm{bp}$ upstream of the transcription start site of FSHB. Nominal evidence for association between endometriosis and SNPs upstream of FSHB was recently reported in independent samples from the UK Biobank providing strong support for this result ${ }^{45}$. FSHB encodes the beta polypeptide of FSH, a glycoprotein hormone that plays a central role in ovarian folliculogenesis ${ }^{46}$. Our index SNP rs74485684 is in high LD with other SNPs located in this region upstream of FSHB including rs11031005 $\left(r^{2}=0.82\right)$ associated with FSH concentrations and rs11031002 $\left(r^{2}=0.64\right)$ associated with LH concentrations ${ }^{24}$. FSH and $\mathrm{LH}$ are related gonadotropin hormones sharing a common alpha subunit. The LH beta subunit is located on chromosome 19 q13.32 and association between these SNPs on chromosome 11 with concentrations for both hormones suggests a common mechanism of regulation. They both play central roles in regulating follicle development in the ovary, influencing oestradiol release during the proliferative phase of the cycle ${ }^{46}$ and contributing to a role for estradiol in endometriosis risk. The allele(s) associated with increased risk of endometriosis are also associated with shorter menstrual cycles, earlier age at menopause, increased dizygotic twinning and polycystic ovarian syndrome ${ }^{24,45,47,48}$.

Our results provide further support for association at the 2p25.1 locus, containing an oestrogen-regulated gene, GREB1, that was first identified in breast cancer cell lines and tumours ${ }^{49}$. In addition to confirming the previously identified association signal at this locus, our results provide evidence for secondary association with risk of endometriosis. Regulation of GREB1 transcription by oestrogen receptor $\alpha(E R \alpha)$ is mediated through three oestrogen response elements (EREs) located $20 \mathrm{~kb}$ upstream of the gene $e^{50,51}$. Moreover, GREB1 functions as an essential component of the oestrogen receptor transcription complex ${ }^{52}$, and while effects of individual risk SNPs are small, the results suggest that risk variants acting on several genes in the same pathway act to increase sensitivity to oestrogen and increase the risk of endometriosis.

This study robustly associated the FN1 locus with endometriosis, in particular with moderate-to-severe disease. Association between rs1250248, which is in very high LD $\left(r^{2}=0.95\right)$ with our lead SNP rs1250241 at this locus, was first reported by the earlier European GWA study led by us, but was not replicated in an independent sample ${ }^{7}$. Results from the current study provide genome-wide evidence for the FN1 locus in Grade B endometriosis, thereby highlighting the importance of subgroup analysis and phenotype definition. FN1 encodes fibronectin, which is a glycoprotein of the extracellular matrix and is also present in plasma, and at the cell surface. Fibronectin is involved in important cellular activities including cell adhesion, growth and migration, and it also plays a critical role in would healing, blood coagulation and metastasis ${ }^{53}$.

Our results provide strong evidence for multiple association signals on chromosome 9p21.3 containing ANRIL (antisense non-coding RNA in the INK4 locus, also known as CDKN2BAS1). A recent Japanese study ${ }^{54}$ showed allele specific effects of rs17761446 on regulation of ANRIL expression with twofold greater chromatin interactions for the protective $G$ allele with the ANRIL promoter. SNP rs17761446 is monomorphic in Europeans and is also in weak LD $\left(r^{2}<0.16\right)$ in Japanese with the SNPs implicated in the current study, suggesting that these associations are independent endometriosis-specific risk loci. SNPs at the chromosome 9p21 locus are also associated with a number of other diseases, including coronary artery disease (CAD) - a disease in which ANRIL has previously been implicated ${ }^{55}$. Chromatin conformation capture in this region in human vascular endothelial cells identified short-range interactions between sequences at the 9p21.3 locus and sequences in the vicinity of the genes encoding CDKN2A, $C D K N 2 B$, and MTAP, and long-range interactions with IFNW1 and IFNA21 approximately one million base pairs upstream on chromosome 9 (ref. 56). Functional studies in mammalian cells show that ANRIL overexpression accelerated proliferation, increased adhesion and decreased apoptosis ${ }^{54,56}$. These functions may be important in endometriosis and hence, additional studies will be necessary to understand how SNPs at this locus in both Japanese and European populations influence endometriosis.

The majority of the identified SNP loci showed larger effects in the Grade B analysis in comparison with those based on analysis of all endometriosis, supporting previous findings of greater genetic loading in moderate-to-severe endometriosis $7,8,57$. The smaller effect sizes in analyses including all endometriosis cases may be due to disease misclassification in self-reported endometriosis cases. However, this would only be part of the explanation. We have previously shown that the genetic contribution to phenotypes of surgically confirmed endometriosis or minimal disease is weaker than for severe disease phenotypes 7,57 . Using polygenic prediction analysis, we also 
showed significant prediction of minimal disease between two independent data sets with surgically confirmed endometriosis ${ }^{57}$.

In summary, this GWA meta-analysis of endometriosis provides evidence for 10 new independent SNP loci, and more than doubles the proportion of genetic variation in endometriosis explained by robust SNP risk loci. These results identify novel variants in or near specific genes with important roles in sex steroid hormone signalling and function, and offer unique opportunities for more targeted functional research efforts.

\section{Methods}

Study overview and GWA Genotyping. Our study included 17,045 endometriosis cases and 191,596 controls from 11 individual case-control data sets of European and Japanese ancestry. The European ancestry arm included 14,926 cases and 189,715 controls from eight individual case-control data sets and the Japanese data sets included 2,119 cases and 2,143 controls from three cohorts. The samples were genotyped on a variety of commercial arrays, as outlined in the Supplementary Information. All samples were collected with informed consent and study protocols were approved by the relevant local institutional ethics committees: the QIMR Human Research Ethics Committee (QIMR), the University of Newcastle and Hunter New England Population Health Human Research Ethics Committees (HCS), the Oxford regional multi-center and local Research Ethics Committees (UK), the Ethical Committees at the Institute of Medical Science at the University of Tokyo and the Center for Genomic Medicine at the RIKEN Yokohama Institute (BBJ), the Commission of Medical Ethics of the Leuven University Hospital (LEUVEN), the Human Subject Committee of Harvard School of Public Health and the Institutional Review Board of Brigham and Women's Hospital (NHS2 and WGHS), the Ethical Committee of the University of Niigata and the affiliated hospitals (Adachi), the Ethical and Independent Review Services (http:// www.eandireview.com) (23andMe), the Danish Research Ethical Committee System (iPSYCH), and the Data Protection Commission of Iceland and the National Bioethics Committee of Iceland (deCODE).

Genome-wide imputation. Genotype data within each case-control data set were subjected to sample and SNP quality control as described in the Supplementary Information. Following a shared protocol, each GWA case-control data set was imputed separately. Imputation was carried out using either minimac ${ }^{58,59}$ (QIMRHCS, LEUVEN, OX, 23andMe, NHS2-dbGaP, BBJ, WGHS and Adachi-500 k), IMPUTE2 (ref. 60) (iPSYCH) or in-house methods ${ }^{9}$ (deCODE). For QIMRHCS, LEUVEN, OX, NHS2-dbGaP, BBJ, WGHS, Adachi-500 K and iPSYCH samples, 1000 Genomes Project March 2012 haplotypes were used as the reference panel, whereas for 23andMe and deCODE samples, 1000 Genomes Project October 2010 haplotypes and whole genome sequence data $(\sim 30$ million sequence variants) of 8,453 Icelanders were used as the reference for imputation, respectively. For the Adachi- 6 data set, individual-level genotype data for all samples were not available and hence no imputation was performed.

\section{Genome-wide association analysis. Imputed genotypes with low imputation} quality ( $<0.3$ for minimac and $<0.4$ for IMPUTE2) in each data set were excluded from the downstream analysis. Association analysis of the imputed (dosage scores or best guess genotypes) or observed genotypes in each case-control data set including all endometriosis cases ('All') was performed using PLINK ${ }^{61}$, SNPTEST $^{62}$ or ProbABEL ${ }^{63}$, assuming an additive model of genetic inheritance. Imputed genotypes in the 23andMe data set were analysed by adjusting for age and the top five principal components, whereas QIMRHCS, LEUVEN, OX, NHS2-dbGaP, BBJ and Adachi-500 K data sets were analysed without any covariates. For Adachi- 6 data set, association analysis was performed using the directly measured genotypes without any covariates. Association analysis of deCODE data was performed using logistic regression ${ }^{9}$, and included adjustment for age and population substructures. The iPSYCH were analysed by adjusting for genotyping waves and the top five principal components.

Genome-wide meta-analysis. The primary meta-analysis of 'All' endometriosis cases versus controls in the 11 individual case-control data sets was performed using the inverse variance-weighted fixed-effect model in $\mathrm{METAL}^{13}$. The $P$ value threshold of $5 \times 10^{-8}$ was declared as genome-wide significant, and SNPs with association at $P<1 \times 10^{-5}$ were considered to show a suggestive association. Heterogeneity of allelic associations was examined using the Cochran's Q statistic ${ }^{64}$ $P<0.05$, as well as the $I^{2}$ index ${ }^{65}$, which indicates the proportion of variance attributable to between-study heterogeneity. Meta-analysis of SNPs associated in the fixed-effect model at $P<1 \times 10^{-5}$ and showing evidence of heterogeneity $(P<0.05)$ was also carried out using the Han Eskin random-effects model $(\text { RE2 })^{17}$ implemented in METASOFT program. Compared to the conventional randomeffects model, the RE2 model offers greater power under heterogeneity. The RE2 model relaxes the conservative assumption in hypothesis testing in the traditional $\mathrm{RE}$ approach and assumes no heterogeneity under the null hypothesis.
Considering the relatively greater genetic loading (liability) of moderate-tosevere (Grade B) endometriosis (rAFS stage III or IV disease) compared to minimal or mild (Grade A) endometriosis (rAFS stage I or II disease or limited ovarian involvement) $)^{7,8,57}$, a secondary analysis was performed for SNPs associated at $P<0.05$, where we performed meta-analysis of Grade B cases versus controls in the QIMRHCS, deCODE, LEUVEN and OX case-control data sets, where disease stage information was readily available.

Per-study Q-Q plots of GWA $P$ values are provided in Supplementary Figs $1-10$ as well as the Q-Q plot for GWA meta-analysis $P$ values (Supplementary Fig. 11). We also provide Q-Q plots for the GWA meta-analysis $P$ values, after excluding the eight previously identified risk loci (Supplementary Fig. 12).

Conditional analysis. We used GCTA ${ }^{18}$ to perform approximate conditional GWA analysis of the newly identified and confirmed risk loci for endometriosis. GCTA allows performing conditional analysis using summary results from GWA meta-analysis and estimated LD from a sufficiently large reference population used in the meta-analysis. Given that the GWA meta-analysis included individuals of both European $(\sim 98 \%)$ and Japanese $(\sim 2 \%)$ ancestries, we used a reference population with similar proportion of European and Japanese individuals to calculate the LD. Best guess genotypes of well-imputed (minimac $r^{2}>0.8$ ) SNPs in the QIMRHCS 1,000 Genomes imputed data were then subjected to quality control to exclude SNPs with Hardy-Weinberg Equilibrium $P<1 \times 10^{-6}$ in controls, $\mathrm{MAF}<0.01$ and $>5 \%$ missingness. As recommended by Yang et al. ${ }^{66}$, samples with estimated relationship score $>0.025$ were also excluded (as opposed to 0.2 used in the meta-analysis), leaving to total of 4,695 samples for further analysis. We then combined the QIMRHCS data set with genotypes of 96 individuals $(\sim 2 \%)$ obtained from the 1000 Genomes Japanese reference data, resulting in 4,791 samples with 7,346,981 autosomal SNPs for LD calculation. To examine if the results were influenced by cross-ancestry LD patterns, we also performed additional conditional analysis using summary statistics from European samples alone and calculated LD from only QIMRHCS samples, and compared the results with those from using both QIMRHCS and the 1000 Genomes Japanese samples.

For each genomic locus (new or confirmed) with $P<5 \times 10^{-8}$, we conservatively searched $\pm 500 \mathrm{~kb}$ surrounding the lead SNP to ensure potential long-range genetic influences were assessed, and adjusted GWA summary data for the lead SNP using the-massoc-cond option in the GCTA ${ }^{18}$. On the basis of genotype data of the reference samples, we first estimated the effective number of independent SNPs within each locus using Genetic type 1 error calculator (GEC) ${ }^{67}$, and then totalled them across all the loci examined for secondary signals. Based on the combined QIMRHCS and 1000 Genomes Japanese reference samples, the effective number of SNPs across all the 14 risk loci was 11,631 , and hence we declared secondary signal if a SNP achieved $P<4.3 \times 10^{-6}(0.05 / 11,631)$.

Where an additional SNP reached threshold for secondary signal after adjustment for the lead SNP, we performed an additional round including both SNPs. If the remaining SNPs had a $P$ value larger than the threshold for secondary signal, no further analysis was performed.

Characterization of endometriosis-associated SNP effects. To examine the effect of potential sources of heterogeneity between groups, we compared effect estimates of our 19 independent risk SNPs for Europeans versus Japanese. To address the effects of disease definition, we also compared the effect estimates of studies with a surgically confirmed diagnosis of endometriosis with those with self-reported endometriosis. A Bonferroni-corrected $P<2.6 \times 10^{-3}$ (corrected for 19 tests) was used to assess statistical significance. Heterogeneity of allelic associations was examined using the Cochran's Q statistic ${ }^{64}$ and evidence of heterogeneity was declared if $P_{\text {het }}<0.05$.

Comparison of identified loci with other traits. We searched the NHGRI GWAS catalogue $^{20}$ for SNP-trait associations, in particular reproductive traits, at our risk loci. SNPs within $500 \mathrm{~kb}$ and in $\mathrm{LD}\left(r^{2}>0.7\right.$; arbitrary number $)$ with the lead SNP at each associated locus were identified using the 1000 Genomes Project pilot 1 genotype data and LD values from CEU population. All the SNPs within each locus were then searched in the NHGRI catalogue (downloaded on 3 March 2016) for genome-wide significant associations with other traits or diseases. Using LD-hub ${ }^{26}$ we also performed LD score regression analysis ${ }^{25}$ to estimate genetic correlation between endometriosis and 199 other traits using summary statistics from fixed-effect meta-analysis including all and Grade B endometriosis cases. A Bonferroni-corrected $P<2.5 \times 10^{-4}$ (corrected for 199 tests) was used to assess statistical significance.

Gene-based association analysis. Gene-based approaches can be more powerful than single SNP analyses, in part due to accounting for allelic heterogeneity (if present) and LD between SNPs, and restricting to genic regions thereby reducing the multiple-testing problem of traditional GWA study. Therefore, using the GWA data from all of the 11 individual case-control data sets, we performed genome-wide gene-based association analysis using VEGAS2 (ref. 28). We first extracted the 4,699,992 SNPs present in all the GWA data sets except Adachi-6 as it includes only observed SNPs and $P$ values from GWA data sets including individuals with European ancestry and the $P$ values from the GWA data sets 
including individuals of Japanese ancestry, and analysed separately using VEGAS2. For each gene, the VEGAS test produces a gene-based $P$ value by incorporating evidence of association from all SNPs across the gene, while accounting for gene size and LD between SNPs. The resulting gene-based $P$ values from GWA studies of European and Japanese ancestry were combined using Stouffer's $Z$-score combined $P$ value method. We tested a total of 22,406 genes (including $10 \mathrm{~kb}$ $5^{\prime}$ and $3^{\prime}$ to their transcriptional start and end positions) with association results for at least two SNPs, and used a Bonferroni-adjusted significance threshold of $P<2.23 \times 10^{-6}(0.05 / 22,406)$ to declare genome-wide significance for gene-based tests.

Fine-mapping analysis. For each independent SNP, we defined a genomic region $500 \mathrm{~kb}$ on either side of the index SNP and computed $99 \%$ credible intervals likely to contain the causal variant using a Bayesian approach, with the strength of evidence for association measured using the Bayes factor for each SNP ${ }^{68,69}$. To assess the resolution of fine-mapping offered by meta-analysis including individuals of both European and Japanese ancestries, we calculated the $99 \%$ credible sets based on GWA meta-analysis results including all studies but Adachi-6, as well as results based on only European studies. We did not compute the $99 \%$ credible sets for the Japanese alone because the small sample size makes comparison of fine-mapping intervals meaningless. We calculated approximate Bayes' factor (BF) for each SNP using:

$$
\mathrm{BF}_{i}=\frac{\sqrt{1-R_{i}}}{\exp \left(-\frac{R \beta_{i}^{2}}{2 \sigma_{i}^{2}}\right)}
$$

where $\beta_{i}$ is the allelic effect of the $i$ th SNP, with corresponding standard error $\sigma_{i}$, and $R_{i}=0.04 /\left(\sigma_{i}^{2}+0.04\right)$, which incorporates a $N\left(0,0.2^{2}\right)$ prior for $\beta_{i}$ assigning high probability to small effect sizes and only small probability to large effect sizes. Adachi-6 data set with only observed SNPs was excluded to maintain uniform SNP coverage across studies. Using the below formula, we calculated the posterior probability that the $i$ th SNP is causal:

$$
\varphi_{i}=\frac{\mathrm{BF}_{i}}{\sum_{i} \mathrm{BF}_{i}}
$$

where the summation in the denominator is over all the SNPs passing quality control across the locus. We assumed a single causal variant for each association signal, calculated BF for all SNPs within $500 \mathrm{~kb}$ of the index SNP, and ranked the SNPs based on their BF. We then constructed a $99 \%$ credible set of variants by combining the ranked SNPs, and calculated the number of SNPs and length of genomic region covered by each credible set.

Identification of putative functional variants. We examined the cis associations between the 19 independent SNPs and other SNPs in high LD $\left(r^{2}>0.7\right)$ with the lead SNPs and expression of nearby genes using the GTEx eQTL portal ${ }^{29}$. A total of 395 SNPs were searched.

We used summary data-based Mendelian randomization $(S M R)^{30}$ to identify potentially causal genes underlying the identified endometriosis associations. Briefly, the SMR method exploits the concept of MR analysis; it tests for the causative effect of an exposure on an outcome using a genetic variant (for example, SNP) as an instrumental variable. In principle, it uses the MR analysis (for example, two-stage least squares approach) to search for causal genes at the loci identified from GWA studies for complex traits. Using summary data from GWA and eQTL studies, the SMR method tests the association between a trait and the expression level of each gene across the whole genome. We used the method to identify causal genes at our endometriosis loci, using the GWA meta-analysis summary results from all studies including all endometriosis cases, and the eQTL summary data from Westra et al. ${ }^{31}$, an eQTL meta-analysis of 5,311 samples from peripheral blood, with SNPs imputed to the HapMap2 reference panels. Of the 14,329 probes in the eQTL data, only probes with at least one cis-eQTL at $P<5 \times 10^{-8}$ were included while excluding probes in the MHC region, resulting in 5,967 probes for final SMR analysis. Therefore, genes with $P<8.4 \times 10^{-6}$ (equivalent to $0.05 / 5,967$ ) were declared to achieve genome-wide significance in the SMR analysis. SMR method also tests this colocalization using the HEIDI (Heterogeneity In Dependent Instruments) test. A $P<0.05$ in the HEIDI test suggests that the majority of the associations identified by the SMR test could be explained by colocalization.

Pathway analysis. We used Data-driven Expression-Prioritized Integration for Complex Traits (DEPICT) ${ }^{32}$ to identify genes and pathways responsible for the observed genetic associations, thereby gaining biological insights at the identified risk loci. Using comprehensive data on gene expression, molecular pathways, experimentally derived protein-protein interactions, phenotypic gene sets, Reactome and KEGG pathways and gene ontology terms, DEPICT highlights the causal genes at each risk locus, enriched pathways and the relevant tissues/cell types where associated genes are highly expressed. Based on the results from the fixed-effect meta-analysis of all the GWA data set, we ran DEPICT analyses on (i) SNPs showing genome-wide significant $\left(P<5 \times 10^{-8}\right)$ association, and (ii) SNPs with suggestive $\left(P<1 \times 10^{-5}\right)$ association signal in GWA meta-analysis including all or Grade B cases.
Variance explained. Based on Neil Risch's liability threshold model ${ }^{19}$, we estimated proportion of variance explained by a single SNP using the effect allele frequency and odds ratio from the GWA meta-analysis of European studies. We used population prevalence of 8 (refs 7,70) and 2.5\% (ref. 71) for all and Grade $\mathrm{B}$ endometriosis cases, respectively.

Assuming associated SNPs are not in high LD, we calculated the sum of single-SNP explained variances to produce the total variance explained by a set of independent SNPs ${ }^{72}$.

Data availability. The authors declare that the data supporting the findings of this study are available within the article and its Supplementary Information Files. For additional data (beyond those included in the main text and Supplementary Information) that support the findings of this study, please contact the corresponding authors.

\section{References}

1. Treloar, S. A., O'Connor, D. T., O'Connor, V. M. \& Martin, N. G. Genetic influences on endometriosis in an Australian twin sample. Fertil. Steril. 71, 701-710 (1999).

2. Gao, X. et al. Economic burden of endometriosis. Fertil. Steril. 86, 1561-1572 (2006).

3. Saha, R. et al. Heritability of endometriosis. Fertil. Steril. 104, 947-952 (2015).

4. Lee, S. H. et al. Estimation and partitioning of polygenic variation captured by common SNPs for Alzheimer's disease, multiple sclerosis and endometriosis. Hum. Mol. Genet. 22, 832-841 (2013).

5. Uno, S. et al. A genome-wide association study identifies genetic variants in the CDKN2BAS locus associated with endometriosis in Japanese. Nat. Genet. 42, 707-710 (2010).

6. Albertsen, H. M., Chettier, R., Farrington, P. \& Ward, K. Genome-wide association study link novel loci to endometriosis. PLoS ONE 8, e58257 (2013).

7. Painter, J. N. et al. Genome-wide association study identifies a locus at $7 \mathrm{p} 15.2$ associated with endometriosis. Nat. Genet. 43, 51-54 (2010).

8. Nyholt, D. R. et al. Genome-wide association meta-analysis identifies new endometriosis risk loci. Nat. Genet. 44, 1355-1359 (2012).

9. Steinthorsdottir, V. et al. Common variants upstream of KDR encoding VEGFR2 and in TTC39B associate with endometriosis. Nat. Commun. 7, 12350 (2016).

10. Sapkota, Y. et al. Independent replication and meta-analysis for endometriosis risk loci. Twin Res. Hum. Genet. 18, 518-525 (2015).

11. Sapkota, Y. et al. Association between endometriosis and the interleukin 1A (IL1A) locus. Hum. Reprod. 30, 239-248 (2014).

12. Rahmioglu, N. et al. Genetic variants underlying risk of endometriosis: insights from meta-analysis of eight genome-wide association and replication datasets. Hum. Reprod. Update 20, 702-716 (2014).

13. Willer, C. J., Li, Y. \& Abecasis, G. R. METAL: fast and efficient meta-analysis of genomewide association scans. Bioinformatics 26, 2190-2191 (2010).

14. Adachi, S. et al. Meta-analysis of genome-wide association scans for genetic susceptibility to endometriosis in Japanese population. J. Hum. Genet. 55, 816-821 (2010).

15. Painter, J. N. et al. Common variants in the CYP2C19 gene are associated with susceptibility to endometriosis. Fertil. Steril. 102, 496-502.e5 (2014).

16. Revised American Society for Reproductive Medicine classification of endometriosis: 1996. Fertil. Steril. 67, 817-821 (1997).

17. Han, B. \& Eskin, E. Random-effects model aimed at discovering associations in meta-analysis of genome-wide association studies. Am. J. Hum. Genet. 88, 586-598 (2011).

18. Yang, J., Lee, S. H., Goddard, M. E. \& Visscher, P. M. GCTA: a tool for genome-wide complex trait analysis. Am. J. Hum. Genet. 88, 76-82 (2011).

19. Risch, N. J. Searching for genetic determinants in the new millennium. Nature 405, 847-856 (2000).

20. Welter, D. et al. The NHGRI GWAS Catalog, a curated resource of SNP-trait associations. Nucleic Acids Res. 42, D1001-D1006 (2014).

21. Pearce, C. L. et al. Association between endometriosis and risk of histological subtypes of ovarian cancer: a pooled analysis of case-control studies. Lancet Oncol. 13, 385-394 (2012).

22. Global Lipids Genetics, C. et al. Discovery and refinement of loci associated with lipid levels. Nat. Genet. 45, 1274-1283 (2013).

23. Lu, X. et al. Genome-wide association study in Han Chinese identifies four new susceptibility loci for coronary artery disease. Nat. Genet. 44, 890-894 (2012).

24. Ruth, K. S. et al. Genome-wide association study with 1000 genomes imputation identifies signals for nine sex hormone-related phenotypes. Eur. J. Hum. Genet. 24, 284-290 (2016).

25. Bulik-Sullivan, B. K. et al. LD Score regression distinguishes confounding from polygenicity in genome-wide association studies. Nat. Genet. 47, 291-295 (2015).

26. Zheng, J. et al. LD Hub: a centralized database and web interface to perform LD score regression that maximizes the potential of summary level GWAS data for SNP heritability and genetic correlation analysis. Bioinformatics 33, 272-279 (2016). 
27. Dunning, A. M. et al. Breast cancer risk variants at $6 \mathrm{q} 25$ display different phenotype associations and regulate ESR1, RMND1 and CCDC170. Nat. Genet. 48, 374-386 (2016)

28. Mishra, A. \& Macgregor, S. VEGAS2: Software for More Flexible Gene-Based Testing. Twin Res. Hum. Genet. 18, 86-91 (2015).

29. Consortium, G. T. Human genomics. The Genotype-Tissue Expression (GTEx) pilot analysis: multitissue gene regulation in humans. Science 348, 648-660 (2015).

30. Zhu, Z. et al. Integration of summary data from GWAS and eQTL studies predicts complex trait gene targets. Nat. Genet. 48, 481-487 (2016).

31. Westra, H. J. et al. Systematic identification of trans eQTLs as putative drivers of known disease associations. Nat. Genet. 45, 1238-1243 (2013).

32. Pers, T. H. et al. Biological interpretation of genome-wide association studies using predicted gene functions. Nat. Commun. 6, 5890 (2015).

33. Nyholt, D. R. et al. Genome-wide association meta-analysis identifies new endometriosis risk loci. Nat. Genet. 44, 1355-1359 (2012).

34. Zondervan, K. T. et al. Beyond Endometriosis Genome-Wide Association Study: from genomics to phenomics to the patient. Semin. Reprod. Med. 34, 242-254 (2016).

35. Kuchenbaecker, K. B. et al. Identification of six new susceptibility loci for invasive epithelial ovarian cancer. Nat. Genet. 47, 164-171 (2015).

36. Powell, J. E. et al. Endometriosis risk alleles at 1p36.12 act through inverse regulation of CDC42 and LINC00339. Hum. Mol. Genet. 25, 5046-5058 (2016).

37. Fung, J. N. et al. Functional evaluation of genetic variants associated with endometriosis near GREB1. Hum. Reprod. 30, 1263-1275 (2015).

38. Holdsworth-Carson, S. J. et al. Endometrial vezatin and its association with endometriosis risk. Hum. Reprod. 31, 999-1013 (2016).

39. Sugino, N., Kashida, S., Karube-Harada, A., Takiguchi, S. \& Kato, H. Expression of vascular endothelial growth factor (VEGF) and its receptors in human endometrium throughout the menstrual cycle and in early pregnancy. Reproduction 123, 379-387 (2002).

40. Brenner, R. M., Nayak, N. R., Slayden, O. D., Critchley, H. O. \& Kelly, R. W. Premenstrual and menstrual changes in the macaque and human endometrium: relevance to endometriosis. Ann. NY Acad. Sci. 955, 60-74 discussion 86-8, 396-406 (2002).

41. Bulun, S. E. et al. Role of estrogen receptor-beta in endometriosis. Semin. Reprod. Med. 30, 39-45 (2012).

42. Burney, R. O. \& Giudice, L. C. Pathogenesis and pathophysiology of endometriosis. Fertil. Steril. 98, 511-519 (2012).

43. Cai, Q. et al. Replication and functional genomic analyses of the breast cancer susceptibility locus at 6q25.1 generalize its importance in women of chinese, Japanese, and European ancestry. Cancer Res. 71, 1344-1355 (2011).

44. Zheng, W. et al. Genome-wide association study identifies a new breast cancer susceptibility locus at 6q25.1. Nat. Genet. 41, 324-328 (2009).

45. Ruth, K. S. et al. Genetic evidence that lower circulating FSH levels lengthen menstrual cycle, increase age at menopause and impact female reproductive health. Hum. Reprod. 31, 473-481 (2016).

46. Gougeon, A. Human ovarian follicular development: from activation of resting follicles to preovulatory maturation. Ann. Endocrinol. (Paris) 71, 132-143 (2010).

47. Jones, M. R. \& Goodarzi, M. O. An update on the genetics of polycystic ovary syndrome: progress and future directions. Fertil. Steril. 106, 25-32 (2016).

48. Mbarek, H. et al. Identification of common genetic variants influencing spontaneous dizygotic twinning and female fertility. Am. J. Hum. Genet. 98, 898-908 (2016).

49. Ghosh, M. G., Thompson, D. A. \& Weigel, R. J. PDZK1 and GREB1 are estrogen-regulated genes expressed in hormone-responsive breast cancer. Cancer Res. 60, 6367-6375 (2000).

50. Deschenes, J., Bourdeau, V., White, J. H. \& Mader, S. Regulation of GREB1 transcription by estrogen receptor alpha through a multipartite enhancer spread over $20 \mathrm{~kb}$ of upstream flanking sequences. J. Biol. Chem. 282, 17335-17339 (2007)

51. Sun, J., Nawaz, Z. \& Slingerland, J. M. Long-range activation of GREB1 by estrogen receptor via three distal consensus estrogen-responsive elements in breast cancer cells. Mol. Endocrinol. 21, 2651-2662 (2007).

52. Mohammed, H. et al. Endogenous purification reveals GREB1 as a key estrogen receptor regulatory factor. Cell Rep. 3, 342-349 (2013).

53. Soikkeli, J. et al. Metastatic outgrowth encompasses COL-I, FN1, and POSTN up-regulation and assembly to fibrillar networks regulating cell adhesion, migration, and growth. Am. J. Pathol. 177, 387-403 (2010).

54. Nakaoka, H. et al. Allelic Imbalance in Regulation of ANRIL through Chromatin Interaction at 9p21 Endometriosis Risk Locus. PLoS Genet. 12, e1005893 (2016).

55. Holdt, L. M. \& Teupser, D. From genotype to phenotype in human atherosclerosis-recent findings. Curr. Opin. Lipidol. 24, 410-418 (2013).

56. Chen, H. H., Almontashiri, N. A., Antoine, D. \& Stewart, A. F. Functional genomics of the 9p21.3 locus for atherosclerosis: clarity or confusion? Curr. Cardiol. Rep. 16, 502 (2014).

57. Sapkota, Y. et al. Genetic burden associated with varying degrees of disease severity in endometriosis. Mol. Hum. Reprod. 21, 594-602 (2015).
58. Li, Y., Willer, C., Sanna, S. \& Abecasis, G. Genotype imputation. Annu. Rev. Genomics Hum. Genet. 10, 387-406 (2009).

59. Li, Y., Willer, C. J., Ding, J., Scheet, P. \& Abecasis, G. R. MaCH: using sequence and genotype data to estimate haplotypes and unobserved genotypes. Genet. Epidemiol. 34, 816-834 (2010).

60. Howie, B. N., Donnelly, P. \& Marchini, J. A flexible and accurate genotype imputation method for the next generation of genome-wide association studies. PLoS Genet. 5, e1000529 (2009).

61. Purcell, S. et al. PLINK: a tool set for whole-genome association and population-based linkage analyses. Am. J. Hum. Genet. 81, 559-575 (2007).

62. Marchini, J., Howie, B., Myers, S., McVean, G. \& Donnelly, P. A new multipoint method for genome-wide association studies by imputation of genotypes. Nat. Genet. 39, 906-913 (2007).

63. Aulchenko, Y. S., Struchalin, M. V. \& van Duijn, C. M. ProbABEL package for genome-wide association analysis of imputed data. BMC Bioinformatics 11, 134 (2010).

64. Cochran, W. G. The Combination of Estimates from Different Experiments. Biometrics 10, 101 (1954).

65. Ioannidis, J. P., Patsopoulos, N. A. \& Evangelou, E. Heterogeneity in metaanalyses of genome-wide association investigations. PLoS ONE 2, e841 (2007)

66. Yang, J. et al. Conditional and joint multiple-SNP analysis of GWAS summary statistics identifies additional variants influencing complex traits. Nat. Genet. 44, 369-375, S1-3 (2012).

67. Li, M. X., Yeung, J. M., Cherny, S. S. \& Sham, P. C. Evaluating the effective numbers of independent tests and significant p-value thresholds in commercial genotyping arrays and public imputation reference datasets. Hum. Genet. 131, 747-756 (2012)

68. Wellcome Trust Case Control, C. et al. Bayesian refinement of association signals for 14 loci in 3 common diseases. Nat. Genet. 44, 1294-1301 (2012).

69. Wakefield, J. A Bayesian measure of the probability of false discovery in genetic epidemiology studies. Am. J. Hum. Genet. 81, 208-227 (2007).

70. Zondervan, K. T., Cardon, L. R. \& Kennedy, S. H. The genetic basis of endometriosis. Curr. Opin. Obstet. Gynecol. 13, 309-314 (2001).

71. Janssen, E. B., Rijkers, A. C., Hoppenbrouwers, K., Meuleman, C. \& D'Hooghe, T. M. Prevalence of endometriosis diagnosed by laparoscopy in adolescents with dysmenorrhea or chronic pelvic pain: a systematic review. Hum. Reprod. Update 19, 570-582 (2013).

72. Replication, D. I. G. et al. Genome-wide trans-ancestry meta-analysis provides insight into the genetic architecture of type 2 diabetes susceptibility. Nat. Genet. 46, 234-244 (2014).

\section{Acknowledgements}

We acknowledge all the study participants in 11 individual endometriosis studies that provided an opportunity for the current study. We also thank many hospital directors and staff, gynaecologists, general practitioners and pathology services in Australia who provided assistance with confirmation of diagnoses. We would like to thank the research participants and employees of 23andMe for making this work possible. We thank the subjects of the Icelandic deCODE study for their participation. We thank research staff and clinicians for providing diagnostic confirmation for the OX data set. We would like to express our gratitude to the staff and members of the Biobank Japan and Laboratory for Statistical Analysis, RIKEN Center for Integrative Medical Sciences for their outstanding assistance. The QIMR study was supported by grants from the National Health and Medical Research Council (NHMRC) of Australia $(241,944,339,462,389,927$, $389,875,389,891,389,892,389,938,443,036,442,915,442,981,496,610,496,739,552,485$ $552,498,1,026,033$ and 1,050,208), the Cooperative Research Centre for Discovery of Genes for Common Human Diseases (CRC), Cerylid Biosciences (Melbourne) and donations from N. Hawkins and S. Hawkins. Analyses of the QIMRHCS and OX GWAS were supported by the Wellcome Trust (WT084766/Z/08/Z) and makes use of WTCCC2 control data generated by the Wellcome Trust Case-Control Consortium (awards 076113 and 085475 ). The iPSYCH study was funded by The Lundbeck Foundation, Denmark (R102-A9118, R155-2014-1724 ), and the research has been conducted using the Danish National Biobank resource supported by the Novo Nordisk Foundation. A full list of the investigators who contributed to the generation of these data is available from http:// www.wtccc.org.uk. D.R.N. was supported in part by the NHMRC Fellowship (613674) and ARC Future Fellowship (FT0991022) schemes. E.G.H. (631096) and G.W.M. $(339446,619667)$ were supported by the NHMRC Fellowships Scheme. S.M. is supported by an Australian Research Council Future Fellowship. A.P.M. was supported by a Wellcome Trust Senior Research Fellowship (award WT098017). N.R. was supported by funding from the Medical Research Council UK (MR/K011480/1). This study was funded by the BioBank Japan project, which is supported by the Ministry of Education, Culture, Sports, Sciences and Technology of Japanese government.

\section{Author contributions}

S.K.L., K.T.Z., S.A.M., T.D., D.I.C., K.S., J.Y.T., G.W.M. and D.R.N. conceived and supervised the study. Y.S., A.P.M., G.W.M. and D.R.N. designed analytical strategies Y.S., A.P.M., A.J.S., S.M., G.T., D.I.C., G.W.M. and D.R.N. performed genome-wide association analysis and imputation. Y.S. performed GCTA conditional analysis, characterization of SNP loci, cross-trait analysis, gene-based analysis, Bayesian 
fine-mapping analysis, pathway and bioinformatic analyses and GWA meta-analyses. F.Z. and J.Y. performed SMR analysis. V.S., A.F., I.D.V., J.E.B., T.L.E., K.M.R., P.M.R. N.G.M., S.A., M.K., L.M.W., D.R.V.E., M.N., K.T.Z., S.A.M., T.D., G.W.M., D.I.C., K.S., J.Y.T. and D.R.N. contributed to GWAS data, sample preparation and clinical phenotyping. A.P.M., L.M.W., D.R.V.E., M.N., K.T.Z., S.A.M., T.D., G.W.M., D.I.C., K.S., J.Y.T. and D.R.N. conducted genotyping and quality control of the data. A.P.M., I.D.V., S.M., N.G.M., D.R.V.E., M.N., K.T.Z., S.A.M., T.D., G.W.M., D.I.C., K.S., J.Y.T. and D.R.N. obtained study funding. Y.S. drafted the manuscript. Y.S., V.S., A.P.M., A.F., N.R., I.D.V., J.E.B., F.Z., T.L.E., S.J., D.O., D.P., K.M.R., P.M.R., A.J.S., S.M., N.G.M., C.M.B., S.A., K.Y., T.E., A.T., Y.K., K.M., M.K., G.T., R.T.G., U.T., L.M.W., J.Y., D.R.V.E., M.N., S.-K.L., K.T.Z., S.A.M., T.D., G.W.M., D.I.C., K.S., J.Y.T. and D.R.N. revised the manuscript and provided final approval.

\section{Additional information}

Supplementary Information accompanies this paper at http://www.nature.com/ naturecommunications

Competing interests: V.S., G.T., U.T. and K.S. are employees of the biotechnology firm deCODE Genetics, a subsidiary of AMGEN. The remaining authors declare no competing financial interests.
Reprints and permission information is available online at http://npg.nature.com/ reprintsandpermissions/

How to cite this article: Sapkota, Y. et al. Meta-analysis identifies five novel loci associated with endometriosis highlighting key genes involved in hormone metabolism. Nat. Commun. 8, 15539 doi: 10.1038/ncomms15539 (2017).

Publisher's note: Springer Nature remains neutral with regard to jurisdictional claims in published maps and institutional affiliations.

\section{(c) (i)}

This work is licensed under a Creative Commons Attribution 4.0 International License. The images or other third party material in this article are included in the article's Creative Commons license, unless indicated otherwise in the credit line; if the material is not included under the Creative Commons license, users will need to obtain permission from the license holder to reproduce the material. To view a copy of this license, visit http://creativecommons.org/licenses/by/4.0/

(C) The Author(s) 2017

\section{iPSYCH-SSI-Broad group}

Thomas M. Werge 24,28,29 and Wesley K. Thompson 24,28,30

${ }^{28}$ Institute of Biological Psychiatry, Mental Health Center, Sct. Hans, Mental Health Services, DK-2100 Copenhagen, Denmark; ${ }^{29}$ Institute of Clinical Sciences, Faculty of Medicine and Health Sciences, University of Copenhagen, DK-2200 Copenhagen, Denmark; ${ }^{30}$ Department of Psychiatry, University of California, San Diego, La Jolla, California 92093, USA. 